\title{
Multiresidue Method for the Rapid Determination of Pesticide Residues in Tea Using Ultra Performance Liquid Chromatography Orbitrap High Resolution Mass Spectrometry and In-Syringe Dispersive Solid Phase Extraction
}

\author{
Hongping Chen, Guanwei Gao, Yunfeng Chai, Guicen Ma, Zhenxia Hao, Chen Wang, Xin Liu,* \\ and Chengyin $\mathrm{Lu}^{*}$ (1)
}

Tea Research Institute, Chinese Academy of Agricultural Sciences, Key Laboratory of Tea Safety \& Control, Key Laboratory of Tea Quality and Safety \& Risk Assessment, Ministry of Agriculture, Hangzhou, 310008, China

\section{Supporting Information}

\begin{abstract}
A method based on in-syringe dispersive solid phase extraction (IS-D-SPE) and ultra performance liquid chromatography Orbitrap high resolution mass spectrometry for the multiresidue analysis of 117 pesticides in tea was developed. Full scan mode was acquired over an $\mathrm{m} / \mathrm{z}$ range of $100-800$ with Orbitrap resolution at 70000, followed by full scan/dd-MS ${ }^{2}$ mode for confirmation. The identification criteria of retention time and mass accuracy tolerance was $\pm 0.20 \mathrm{~min}$ and $\pm 5.0 \mathrm{ppm}$, respectively. MS/MS fragment ions obtained dd-MS ${ }^{2}$ were necessary to identify the pesticides with the same molecular mass weight. The IS-D-SPE technique involved a mixture of $200 \mathrm{mg}$ PSA, $100 \mathrm{mg} \mathrm{C} 18$, and $15 \mathrm{mg}$ multiwalled carbon nanotubes for the cleanup of tea matrix. Good linearity $\left(R^{2}>0.99\right)$ for 117 pesticides was obtained. Satis-

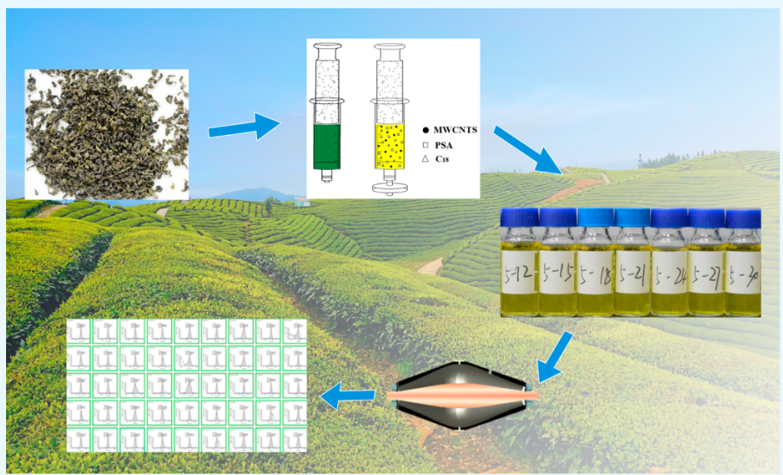
factory recoveries in the range of $70-120 \%$ were obtained for 105 pesticides, while intraday and interday precisions were below $20 \%$. Limits of quantification were generally $10 \mu \mathrm{g} \mathrm{kg}^{-1}$. Finally, this method was employed to analyze 117 pesticides in 70 tea samples.
\end{abstract}

\section{INTRODUCTION}

Tea is the most widely consumed beverage next to water in the world. By drinking a cup of tea, one can take in plenty of special nutrient substances, such as catechins, theanine, polysaccharide, vitamins, and essential minerals. These substances are beneficial for human health due to their effective medicinal and therapeutic potentialities, such as cancer prevention, antioxidants, anticardiovascular diseases, correcting skin disorder, and body weight reduction. ${ }^{1}$ Besides beneficial healthy function, the fascinating aroma and comfortable taste attract people to enjoy tea, especially young people.

Tea (Camellia sinensis L) is a perennial woody plant and is grown in regions such as tropical, subtropical, and temperature zones. These growth conditions are favorable for pests, diseases, and competing grasses. In China, more than 800 pests and diseases have been found in tea gardens, which results in serious damage of tea yield and quality. ${ }^{2}$ Currently, application of pesticide formulations is the most effective measure to prevent pests and disease, and improve tea yield and quality. Regarding the negative effects of the application of pesticides in tea, there are potentially harmful residues. The presence of pesticide is an important issue in tea safety and tea trade. ${ }^{3}$ Many countries and regions, such as Japan, European Union (EU), China, and Codex Alimentarius Commission (CAC), have formulated maximum residue levels (MRLs) in tea. The strictest MRLs are set by the EU, where 515 pesticide MRLs are regulated, and MRLs for unregulated pesticides are set at $0.010 \mathrm{mg} \mathrm{kg}^{-1}$. Japan also has set up 264 pesticide MRLs and the unregulated pesticide MRLs are at $0.01 \mathrm{mg} \mathrm{kg}^{-1} .5$ Chinese national standard has formulated 387 pesticide MRLs in agricultural products, where 48 pesticide MRLs in tea have been regulated in the range of $0.1-20 \mathrm{mg} \mathrm{kg}^{-1}$. Therefore, high throughput pesticide screening at trace levels is necessary to ensure tea safety and reduce the tea economic loss due to exceeding the pesticide MRLs.

Chromatography coupled with mass spectrometry is widely used for the screening and determination of pesticide residues in tea. ${ }^{7,8}$ Gas chromatography coupled with single mass spectrometry (GC-MS) or tandem mass spectrometry (GC-MS/ MS) is employed for the analysis of volatile and thermostable pesticides, while liquid chromatography tandem mass spectrometry (HPLC-MS/MS) is alternatively used to detect the polar, nonvolatile, and thermally unstable pesticides. ${ }^{9}$ A high throughput GC-MS and HPLC-MS/MS method for the multiresidue

Received: June 29, 2017

Accepted: August 30, 2017

Published: September 18, 2017 

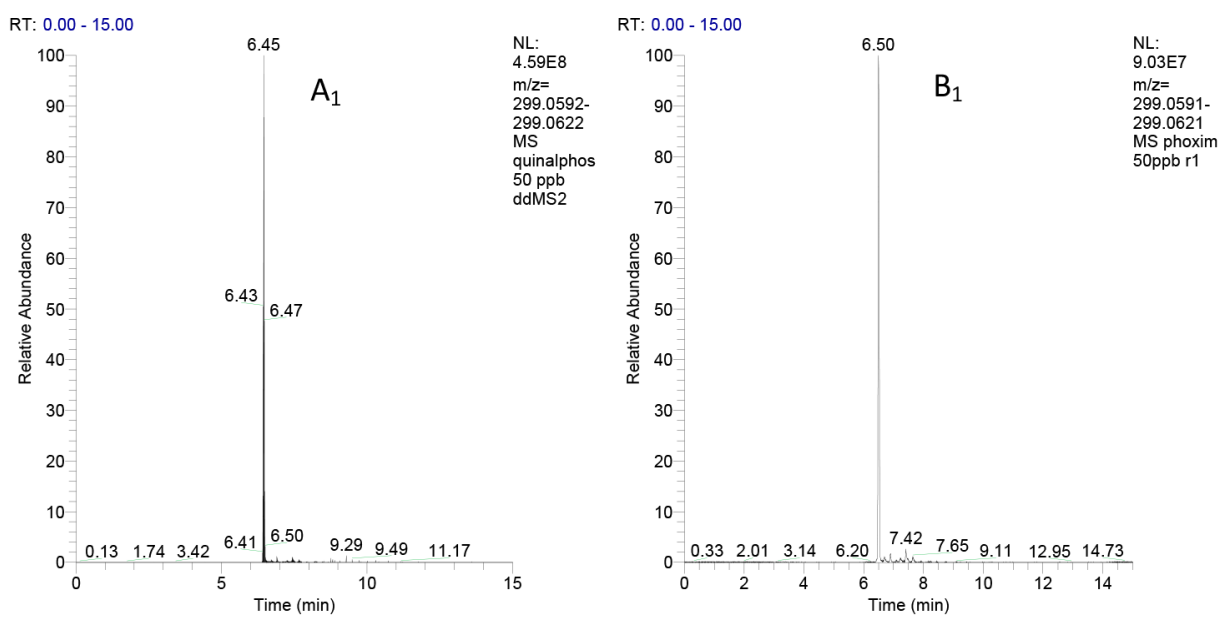

quinalphos 50 ppb ddMS2 \#2749 RT: 6.46 AV: $1 \quad \mathrm{NL}: 3.98 \mathrm{E} 8$

phoxim 50ppb r1 \#2791 RT: 6.50 AV: 1 NL: $8.65 E 7$
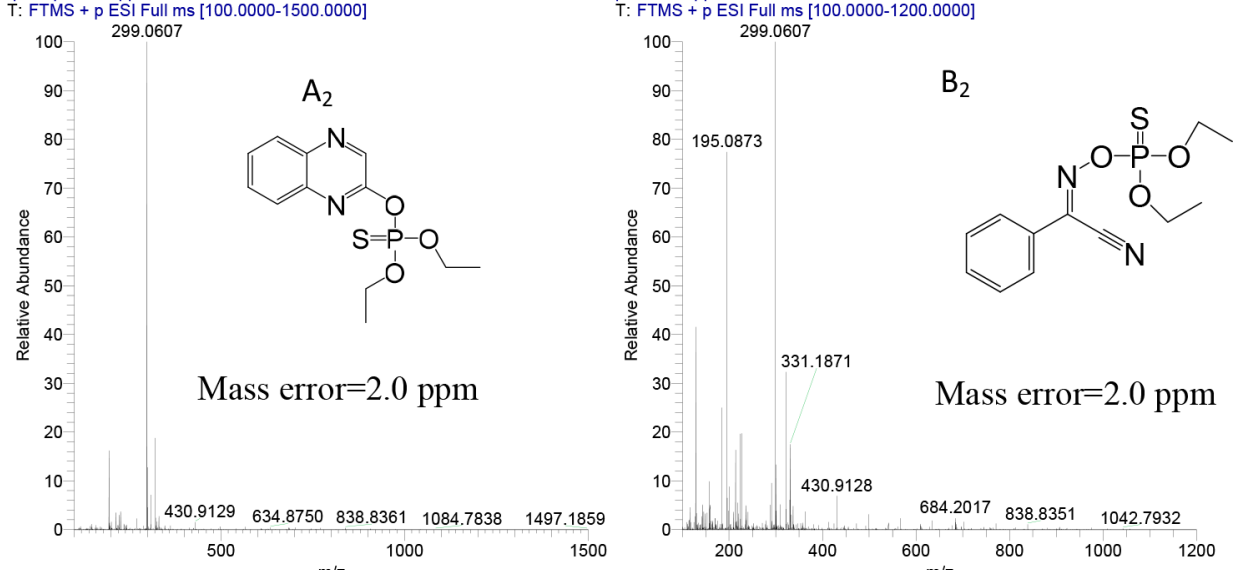

quinalphos 50 ppb ddMS2 \#2664-2794 RT: $6.26-6.56$ AV: 3 , T: Average spectrum MS2 299.16 (2664-2794)

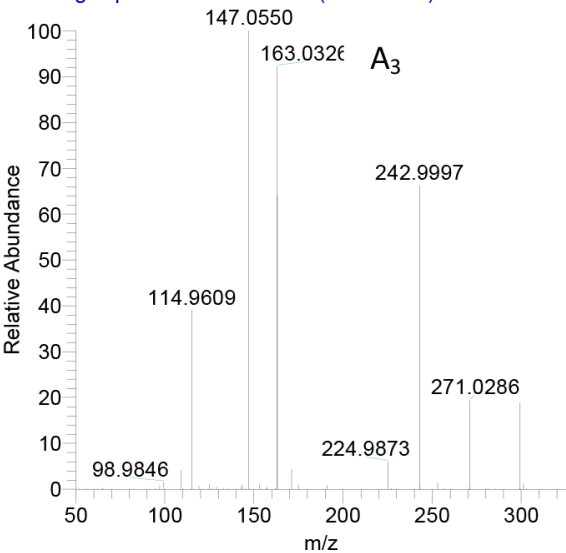

3 phoxim 50ppb ddMS2 \#2709-3262 RT: $6.38-7.68$ AV: 3 NL:

T: Average spectrum MS2 299.16 (2709-3262)

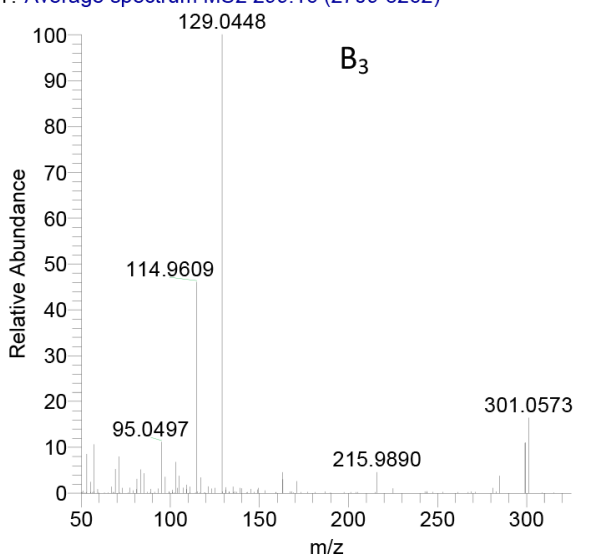

Figure 1. Extracted ion chromatograms $(m / z 299.0607$ with exact mass error 5 ppm $)\left(A_{1}, B_{1}\right)$, full scan mass spectrogram $\left(A_{2}, B_{2}\right)$, and dd-MS fragmented ions $\left(\mathrm{A}_{3}, \mathrm{~B}_{3}\right)$ for quinalphos $(\mathrm{A})$ and phoxim $(B)$ in standard solution at $50 \mu \mathrm{g} \mathrm{L}^{-1}$.

determination of 490 and 448 pesticides, respectively, has been developed and validated by Pang et al. ${ }^{10}$ For GC-MS/MS and HPLC-MS/MS, several parameters, such as the precursor and two MS/MS transitions, retention time, and the area ratio between two MS/MS transitions, are required to be monitored. However, false positive or negative findings for the MS/MS technique may occur due to the use of nonspecific transitions, characterized by a poorly resolved chromatographic peak. ${ }^{11}$ Other drawbacks and limitations for the MS/MS technique which are inherent to targeted acquisition are as follows: timeconsuming optimization of MS/MS parameters, limited number of compounds in one instrumental method, time-consuming and constant definition of acquisition-time window, and nonretrospective data analysis. ${ }^{12}$ Although LC-MS/MS and GC-MS/ MS employing triple-quadrupole MS systems are traditionally used for multipesticide analysis, the use of high resolution mass spectrometry (HRMS) with full-scan technique has become a promising analytical technique for the high throughput screening and determination of organic pollutants in foods, the environment, and other matrices. ${ }^{13,14}$ Compared with MS/MS, the HRMS technique has several advantages that are important for screening and identification of unknown compounds: higher sensitivity, higher accurate mass for calculating elemental composition, and higher accurate mass of product ions $\left(\mathrm{MS}^{\mathrm{n}}\right)$. $^{15}$ 

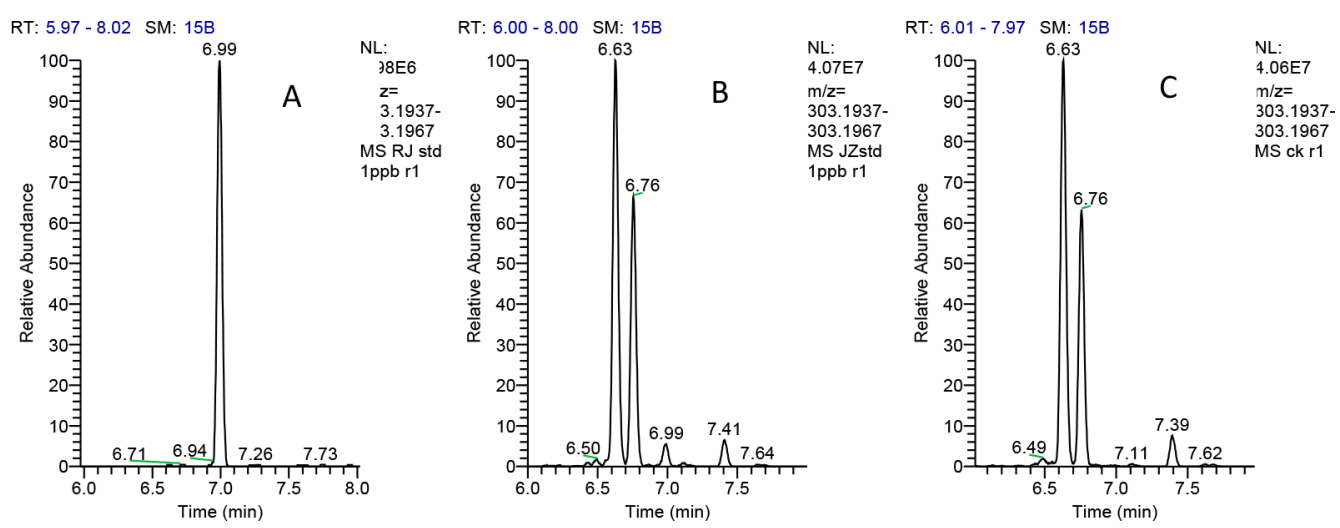

Figure 2. Extracted ion chromatograms (mass error $5 \mathrm{ppm}$ ) for allethrin $(\mathrm{m} / z 303.1952$, RT $6.99 \mathrm{~min})$ at $1 \mu \mathrm{g} \mathrm{L}^{-1}$ in methanol (A), green tea matrix matched calibrated solution (B), and blank green tea matrix (C).

The Orbitrap high resolution mass analyzer was first described in 2000 and commercially introduced in $2005 .^{16,17}$ In Orbitrap HRMS, the option for an internal mass calibration can be chosen to correct all the scans in a LC-Orbitrap MS experiment. ${ }^{18} \mathrm{~A}$ mass accuracy greater than $5 \mathrm{ppm}$ at resolving power $60,000 \mathrm{fwhm}$ (full-width at half-maximum peak height at $m / z 400$ ) can be achieved due to the use of the Orbitrap mass analyzer preceded by an external injection device based on trapping ions RF-only gas-filled curved quadrupole (named C-trap). ${ }^{19}$ Due to accurate mass and high sensitivity, the utility of HPLC-Orbitrap-MS is sufficient for the measurement of a wide range of pesticides at trace residue in various matrices, such as fruit and vegetables, ${ }^{20}$ honey, ${ }^{21}$ fish, ${ }^{22}$ and baby foods. ${ }^{23}$ Currently, there are few literature references on this analytical technique for high throughput screening pesticide residues in tea using HPLC Orbitrap MS. Until now, only two studies related to high throughput screening pesticide residues in green tea and its nutraceuticals employing HPLC Orbitrap MS have been reported. One previous report focused on screening pesticide residues in the nutraceutical products, such as tea powder and extracts. ${ }^{24}$ The tea nutraceuticals are generated from raw tea leaves or manufactured tea and would have undergone complex processing. The presence of matrix differences may result in different matrix interference and method performance when HPLC Orbitrap MS is applied. Another previous study focused on the UPLC-Q-Orbitrap MS identification of pesticide residues in agricultural products, such as vegetables, fruits, and green tea. ${ }^{25}$ Therefore, it is necessary to establish a novel methodology for screening and determination of multipesticide residues in tea using UPLC-Q-Orbitrap MS.

The purpose of this study was to develop a high throughput screening and multi residue analysis of pesticides in various tea samples (green tea, black tea, and oolong tea) using UPLC-QOrbitrap-MS. A rapid and simple sample preparation employing in-syringe dispersive solid phase extraction (IS-D-SPE) cleanup was optimized. Full scan/dd-MS ${ }^{2}$ monitoring mode was used for screening and identification of targeted pesticides.

\section{EXPERIMENTAL SECTION}

2.1. Chemicals and Reagents. All pesticides were of certified quality and purchased from Dr. Ehrenstorfer (Augsburg, Germany), Sigma-Aldrich (Steinheim, Germany), J\&K Chemical (Beijing, China). Full name, chemical formula, and accurate mass are listed in Supporting Information Table-S1. HPLC-grade acetonitrile, methanol, and acetone were provided from SigmaAldrich (Merck, Germany). HPLC-MS grade formic acid and ammonium acetate were purchased from Sigma-Aldrich. Deionized water was obtained using a Milli-Q plus ultrapure water system from Millipore (Miford, USA). The sorbents of primary second amine (PSA), multiple walled carbon nanotubes (MWCNTs), and octadecylsilane (C18) were provided from Agela (Tianjin, China). Analytical-grade sodium chloride and anhydrous magnesium were provided from Zhejiang Medicine (Hangzhou, China), and baked for $3 \mathrm{~h}$ at $650{ }^{\circ} \mathrm{C}$ prior to use.

2.2. Samples. All tea samples (green tea, black tea, and oolong tea) were obtained from Laboratory of Tea Safety and Risk Assessment, Ministry of Agriculture, P.R. China. Organic tea samples free of pesticides were used for matrix standard solution and recovery test. Ten positive tea samples, which were previously detected and confirmed using UPLC-MS/MS, were employed for the optimization of the extraction procedure. All tea samples were stored in a dark room at $1-4{ }^{\circ} \mathrm{C}$.

2.3. Standard Solution Preparation. Individual stock standard solutions (generally at $1000 \mu \mathrm{g} \mathrm{mL}^{-1}$ ) were prepared in methanol or dissolved in a few volumes of acetone and then added to the scale of volumetric flask using methanol. Of these stock solutions, standard mixture solutions were diluted at $5 \mu \mathrm{g} \mathrm{mL}^{-1}$ using methanol. Working standard solutions were prepared by the serial dilution of standard mixture solutions using methanol. Matrix matched standard solutions were prepared by adding less than or equal to $100 \mu \mathrm{L}$ of working standard solution into about $900 \mu \mathrm{L}$ of the blank extractions (total volume of standard solution and blank extraction was

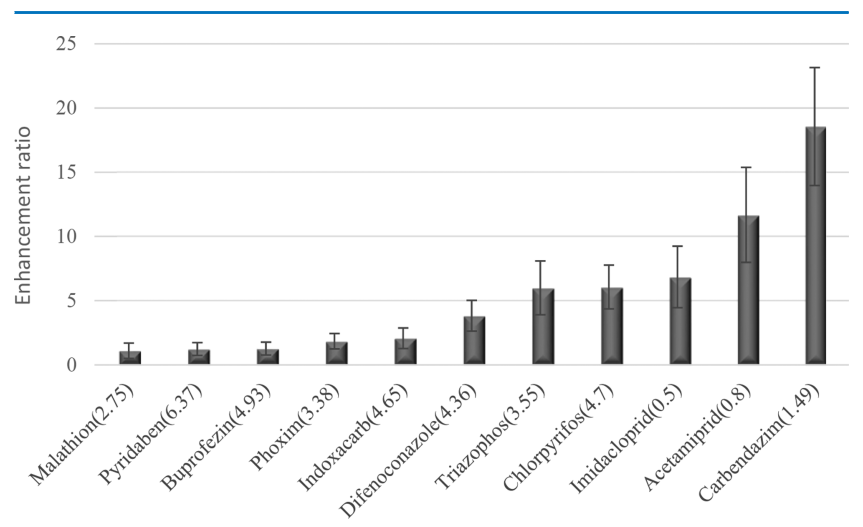

Figure 3. Enhancement of extraction efficiency of 11 representative pesticides (values of Log $K_{\mathrm{ow}}$ are given in brackets) in incurred tea samples with or without water soaking prior acetonitrile extraction $(n=5)$. 


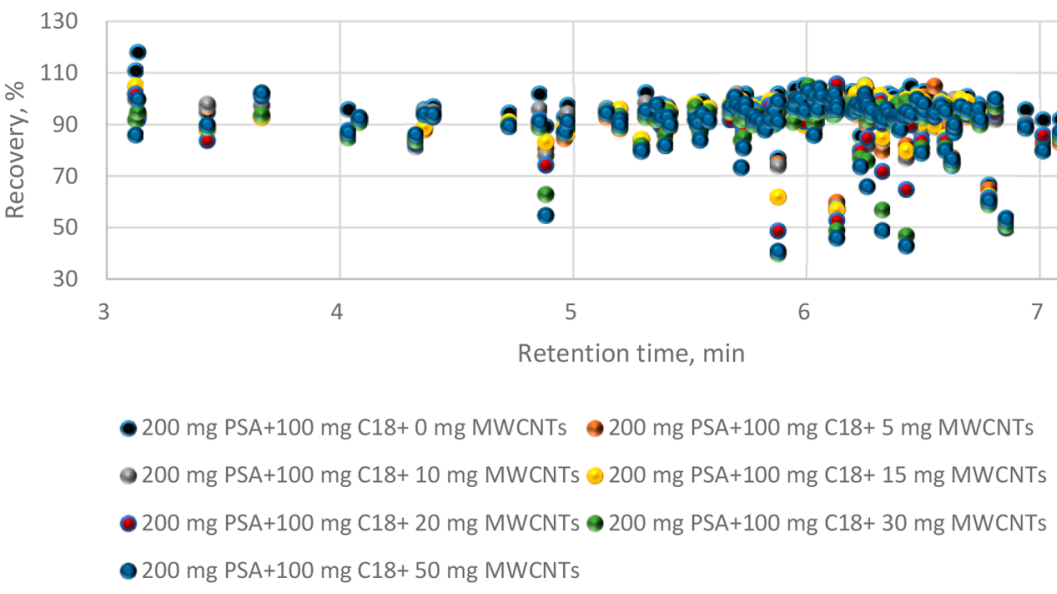

Figure 4. Recovery of 117 pesticides with $200 \mathrm{mg}$ PSA, $100 \mathrm{mg} \mathrm{C18}$, and different amounts of MWCNTs for cleanup of the tea matrix.

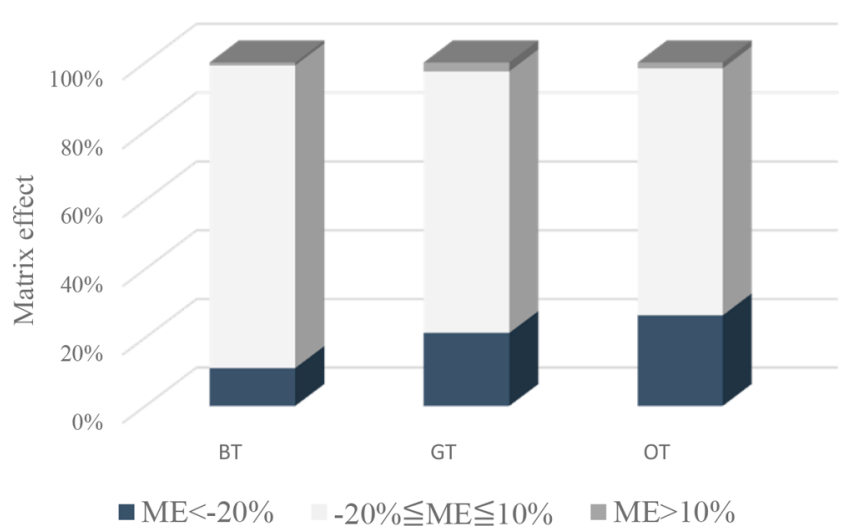

Figure 5. Evaluation of matrix effect in black tea (BT), green tea (GT), and oolong tea (OT).

equal to $1 \mathrm{~mL}$ ), which were obtained from organic tea samples and cleaned up by IS-D-SPE.

2.4. Sample Preparation. The proposed sample preparation method was performed using acetonitrile extraction by vortex and IS-D-SPE cleanup. Homogenized tea powder $(2.5 \mathrm{~g})$ was weighed into a $50 \mathrm{~mL}$ centrifuged tube, and then $5 \mathrm{~mL}$ of deionized water was added and mixed by a vortex at $1200 \mathrm{rpm}$ for $1 \mathrm{~min}$. Ten $\mathrm{mL}$ of acetonitrile was added, followed by extraction using a vortex at $1200 \mathrm{rpm}$ for $2 \mathrm{~min}$. Afterward, $2 \mathrm{~g}$ of $\mathrm{NaCl}$ and $4 \mathrm{~g}$ of $\mathrm{MgSO}_{4}$ were added and mixed by a vortex at $1200 \mathrm{rpm}$ for $1 \mathrm{~min}$, and followed by centrifugation at $5000 \mathrm{rpm}$ for $5 \mathrm{~min}$. Two $\mathrm{mL}$ of upper layer extraction was transferred into a syringe containing $200 \mathrm{mg}$ PSA, $100 \mathrm{mg} \mathrm{C18}$, and $15 \mathrm{mg}$ MWCNTs. After plugging the bottom, the syringe was blended using a vortex at $1200 \mathrm{rpm}$ for $1 \mathrm{~min}$. Finally, the plug was changed with a $0.22 \mu \mathrm{m}$ filter membrane. The extraction was transferred into sample vial for UPLC-Q-Orbitrap-MS analysis.

To determine the effect of water immersion on extraction efficiency, the incurred samples were extracted with acetonitrile with and without water immersion. Comparison of different extraction methods, e.g., stand by for overnight, homogenizer, vortex, and shanking device, were carried out for shortening extraction time and streamlining sample preparation.

2.5. UPLC-Q-Orbitrap-MS Parameters. UltiMate 3000 HPLC system (Thermo Fisher Scientific, Waltham,MA, USA) was used for the separation of the analytes on the reverse phase Hypersil GOLD C18 $(100 \mathrm{~mm} \times 2.1 \mathrm{~mm}$ i.d., $3 \mu \mathrm{m}$ particle size, (Thermo Fisher Scientific, Waltham,MA, USA). The mobile phase consisted of $0.1 \%(\mathrm{v} / \mathrm{v})$ formic acid and ammonium formate $4 \mathrm{mM}$ in water (A) and methanol (B). A gradient elution was applied as follows: $10 \% \mathrm{~B}, 0-1 \mathrm{~min} ; 10-75 \% \mathrm{~B}$, 1-3 min; $75-100 \% \mathrm{~B}, 3-4 \mathrm{~min} ; 100 \% \mathrm{~B}, 4-10 \mathrm{~min} ; 100-10 \% \mathrm{~B}$, 10-11 $\mathrm{min} ; 10 \% \mathrm{~B}, 11-14 \mathrm{~min}$. The sample tray temperature, column oven temperature, injection volume, and flow rate set at $20{ }^{\circ} \mathrm{C}, 30{ }^{\circ} \mathrm{C}, 10 \mu \mathrm{L}$, and $0.3 \mathrm{~mL} \mathrm{~min}^{-1}$, respectively.

The UPLC system was coupled with a Q-Orbitrap MS, a high-performance benchtop quadrupole Orbitrap mass spectrometer (Thermo Fisher Scientific, Waltham,MA, USA). Ionization was achieved by operating a heated electrospray ionization (H-ESI) probe in the positive mode. The Orbitrap MS parameters were as follows: sheath gas $\left(\mathrm{N}_{2},>99 \%\right), 35 \mathrm{~mL}$; auxiliary gas $\left(\mathrm{N}_{2},>99 \%\right), 10 \mathrm{~mL}$; heater temperature, $350{ }^{\circ} \mathrm{C}$; capillary temperature, $300{ }^{\circ} \mathrm{C}$; spray voltage, $4 \mathrm{kV}$; skimmer voltage, $18 \mathrm{~V}$; capillary voltage, $35 \mathrm{~V}$; tube lens voltage, $95 \mathrm{~V}$. Quantification and screening data were acquired using full scan and full scan/dd-MS ${ }^{2}$ mode, respectively. The full scan mode was acquired over an $\mathrm{m} / \mathrm{z}$ range of $100-800$ with Orbitrap resolution at 70000 full width at half-maximum (fwhm) at $m / z=200$. Automatic gain control (AGC) target ion set at $1.0 \mathrm{e}^{6}$ for a maximum injection time of $100 \mathrm{~ms}$. Stepped normalized collision energies (NCEs) were set at $30,50,70 \mathrm{eV}$. The dd-MS ${ }^{2}$ resolution was set at $17500 \mathrm{fwhm}(\mathrm{m} / z=200)$. The precursor ions with a signal threshold $>1.0 \mathrm{e}^{4}$ were automatically performed for $\mathrm{MS}^{2}$ fragmentation activation with $40 \%$ collision energy.

2.6. Data Processing. Data acquisition and processing were performed using Tracefinder 3.3 software package. Data calculations and statistical analysis were performed using Microsoft Excel and SPSS 12.0, respectively. Accurate-mass database of protonated molecular ion $[\mathrm{M}+\mathrm{H}]^{+}$or ammoniated molecular ion $\left[\mathrm{M}+\mathrm{NH}_{4}\right]^{+}$and $\mathrm{MS} / \mathrm{MS}$ fragment ions, and retention time were obtained by injection of individual standard solutions at $500 \mathrm{ng} \mathrm{mL}^{-1}$. The defined criteria for identification analysis was as follows: retention time $\pm 0.20 \mathrm{~min}$, mass accuracy tolerance $\pm 5.0 \mathrm{ppm}$, identical molecular ions, and MS/MS fragment ions. The most abundant ion, typically the protonated molecular or ammoniated molecular ion, was used for quantification.

2.7. Validation Procedure. Matrix effect was evaluated by comparing the slopes of the analytical curves obtained using matrix matched standards and solvent. Each analytical curve was established at six different concentrations in the range of 
Table 1. Recoveries $\%(n=5)$ and Precision $^{a}$ of 117 Pesticides from Black Tea, Green Tea, and Oolong Tea

\begin{tabular}{|c|c|c|c|c|c|c|c|c|}
\hline \multirow[b]{3}{*}{ number } & \multirow[b]{3}{*}{ pesticide } & \multicolumn{6}{|c|}{ level of fortification, $\mu \mathrm{g} \mathrm{kg}^{-1}$} & \multirow[b]{3}{*}{ interday RSD,\% } \\
\hline & & \multicolumn{2}{|c|}{ black tea } & \multicolumn{2}{|c|}{ green tea } & \multicolumn{2}{|c|}{ oolong tea } & \\
\hline & & 10 & 50 & 10 & 50 & 10 & 50 & \\
\hline 1 & Acetamiprid & $83(9.5)$ & $88(5.6)$ & $75(11.5)$ & $78(3.2)$ & $83(6.3)$ & $86(5.9)$ & 9.6 \\
\hline 2 & Acetochlor & $94(8.7)$ & $96(3.3)$ & $92(7.9)$ & $88(3.1)$ & $87(10.6)$ & $91(5.7)$ & 6.7 \\
\hline 3 & Allethrin & $94(14.7)$ & $92(9.7)$ & $81(8.9)$ & $88(6.3)$ & $85(11.6)$ & $93(8.5)$ & 10.5 \\
\hline 4 & Ametryn & $89(6.3)$ & $94(4.7)$ & $78(3.2)$ & $83(5.1)$ & $87(7.6)$ & $84(5.2)$ & 4.8 \\
\hline 5 & Atraton & $85(5.5)$ & $87(4.9)$ & $81(7.1)$ & $82(6.8)$ & $78(8.8)$ & $86(7.3)$ & 6.2 \\
\hline 6 & Atrazine & $92(3.6)$ & $96(5.1)$ & $82(5.6)$ & $90(7.3)$ & $85(2.5)$ & $93(4.7)$ & 4.9 \\
\hline 7 & Azoxystrobin & $95(4.6)$ & $91(2.7)$ & $92(1.8)$ & $94(3.2)$ & $85(3.2)$ & $95(1.9)$ & 3.7 \\
\hline 8 & Buprofezin & $87(2.1)$ & $90(1.7)$ & $89(5.4)$ & $87(7.3)$ & $93(3.4)$ & $94(4.1)$ & 1.8 \\
\hline 9 & Butachlor & $87(1.8)$ & $91(0.7)$ & $86(3.2)$ & $76(5.6)$ & $82(1.7)$ & $81(3.6)$ & 2.8 \\
\hline 10 & Carbaryl & $83(14.6)$ & $87(7.5)$ & $94(16.3)$ & $83(10.5)$ & $90(13.7)$ & $79(7.6)$ & 9.7 \\
\hline 11 & Carbendazim & $68(6.2)$ & $73(4.8)$ & $66(6.9)$ & $69(7.5)$ & $68(8.1)$ & $73(7.6)$ & 5.9 \\
\hline 12 & Carbofuran & $81(2.3)$ & $93(3.1)$ & $85(1.7)$ & $90(1.9)$ & $78(4.3)$ & $84(3.8)$ & 3.8 \\
\hline 13 & Chlorantraniliprole & $84(10.7)$ & $88(5.6)$ & $76(9.8)$ & $73(7.5)$ & $80(6.9)$ & $84(5.7)$ & 8.2 \\
\hline 14 & Chlorbenzuron & $21(15.8)$ & $26(8.8)$ & $28(16.3)$ & $30(7.5)$ & $31(13.9)$ & $27(6.8)$ & 8.6 \\
\hline 15 & Chlorfluazuron & $61(12.7)$ & $65(6.4)$ & $61(11.6)$ & $71(7.0)$ & $65(9.9)$ & $63(6.3)$ & 9.1 \\
\hline 16 & Chlorotoluron & $46(7.9)$ & $49(8.5)$ & $52(7.5)$ & $47(6.2)$ & $48(7.2)$ & $49(6.7)$ & 8.1 \\
\hline 17 & Chlorpyrifos & $85(8.6)$ & $82(5.1)$ & $80(2.8)$ & $84(2.6)$ & $77(1.9)$ & $86(1.1)$ & 3.0 \\
\hline 18 & Chlorpyrifos-methyl & $88(8.5)$ & $91(4.3)$ & $89(7.6)$ & $96(4.1)$ & $80(3.6)$ & $85(5.3)$ & 6.7 \\
\hline 19 & Chromafenozide & $92(9.6)$ & $97(3.4)$ & $83(7.5)$ & $92(8.6)$ & $92(9.7)$ & $94(4.2)$ & 8.4 \\
\hline 20 & Clofentezine & $22(8.9)$ & $27(6.8)$ & $32(9.7)$ & $34(6.1)$ & $25(11.6)$ & $23(6.6)$ & 9.3 \\
\hline 21 & Coumphos & $82(1.7)$ & $84(2.5)$ & $90(3.0)$ & $96(2.1)$ & $85(0.8)$ & $89(3.1)$ & 2.7 \\
\hline 22 & Cyanazine & $80(9.4)$ & $83(6.2)$ & $84(8.5)$ & $87(7.7)$ & $78(6.9)$ & $80(7.8)$ & 7.5 \\
\hline 23 & Diazinon & $89(0.8)$ & $93(1.5)$ & $92(2.7)$ & $95(1.5)$ & $79(1.7)$ & $86(2.4)$ & 2.4 \\
\hline 24 & Dichlorvos & $83(8.5)$ & $88(4.9)$ & $86(7.6)$ & $95(6.4)$ & $81(6.9)$ & $89(7.8)$ & 8.6 \\
\hline 25 & Difenoconazole & $95(2.3)$ & $92(2.7)$ & $93(3.9)$ & $91(2.1)$ & $88(4.2)$ & $90(5.9)$ & 4.3 \\
\hline 26 & Diflubenzuron & $40(6.7)$ & $42(5.3)$ & $54(8.1)$ & $57(9.4)$ & $41(7.6)$ & $53(7.8)$ & 8.1 \\
\hline 27 & Diflufenican & $48(7.9)$ & $59(4.7)$ & $64(6.3)$ & $61(5.1)$ & $43(8.3)$ & $49(3.7)$ & 8.9 \\
\hline 28 & Dimetachlone & $86^{b}(9.7)$ & $92(7.6)$ & $90^{b}(11.6)$ & $95(7.9)$ & $87^{b}(14.3)$ & $95(9.8)$ & 7.7 \\
\hline 29 & Dimethachlor & $93(3.7)$ & $91(2.6)$ & $79(4.8)$ & $90(1.5)$ & $91(5.0)$ & $88(1.7)$ & 2.9 \\
\hline 30 & Dimethenamid & $90(1.1)$ & $95(2.0)$ & $87(2.5)$ & $92(1.4)$ & $90(2.4)$ & $93(1.7)$ & 3.2 \\
\hline 31 & Dimethoate & $82(5.7)$ & $85(4.6)$ & $79(6.2)$ & $80(4.1)$ & $80(6.7)$ & $82(3.9)$ & 5.9 \\
\hline 32 & Dimethomorph & $100(8.5)$ & $97(3.5)$ & $101(6.1)$ & $99(4.9)$ & $99(5.8)$ & $100(4.2)$ & 7.1 \\
\hline 33 & Diuron & $87(8.6)$ & $85(3.5)$ & $72(9.6)$ & $85(3.7)$ & $83(8.3)$ & $78(5.2)$ & 6.8 \\
\hline 34 & Epoxiconazole & $89(1.1)$ & $91(3.2)$ & $92(4.6)$ & $95(2.7)$ & $89(5.7)$ & $87(3.2)$ & 4.6 \\
\hline 35 & Ethiofencarb & $87(16.4)$ & $90(5.3)$ & $79(9.8)$ & $72(6.8)$ & $82(15.7)$ & $87(9.6)$ & 8.7 \\
\hline 36 & Ethion & $95(3.2)$ & $95(4.7)$ & $93(1.8)$ & $86(2.5)$ & $82(4.6)$ & $84(1.9)$ & 3.3 \\
\hline 37 & Ethoprop & $96(1.7)$ & $95(0.8)$ & $93(2.3)$ & $96(3.1)$ & $92(2.8)$ & $96(1.8)$ & 2.9 \\
\hline 38 & Fenazaquin & $57(5.3)$ & $53(2.1)$ & $58(2.2)$ & $61(4.3)$ & $49(2.7)$ & $57(1.7)$ & 4.1 \\
\hline 39 & Fenobucarb & $89(1.8)$ & $95(1.1)$ & $93(2.4)$ & $98(1.6)$ & $94(3.2)$ & $93(1.8)$ & 3.7 \\
\hline 40 & Flonicamid & $72(4.3)$ & $82(5.1)$ & $79(2.9)$ & $77(7.4)$ & $80(6.3)$ & $76(2.1)$ & 6.5 \\
\hline 41 & Flufenacet & $93(8.6)$ & $96(4.3)$ & $92(9.6)$ & $90(3.9)$ & $91(9.6)$ & $89(7.9)$ & 8.2 \\
\hline 42 & Flusilazole & $91(2.3)$ & $97(5.4)$ & $97(4.8)$ & $100(1.9)$ & $94(5.3)$ & $96(4.9)$ & 4.7 \\
\hline 43 & Fonofos & $88(2.7)$ & $104(3.2)$ & $91(1.8)$ & $98(2.5)$ & $96(2.5)$ & $91(1.6)$ & 3.7 \\
\hline 44 & Furalaxyl & $86(1.3)$ & $94(2.7)$ & $84(0.9)$ & $88(1.5)$ & $90(3.2)$ & $88(1.6)$ & 3.2 \\
\hline 45 & Hexaconazole & $83(2.8)$ & $90(3.1)$ & $90(5.4)$ & $92(2.9)$ & $91(6.4)$ & $89(1.8)$ & 4.8 \\
\hline 46 & Hexazinone & $79(7.5)$ & $90(7.1)$ & $82(3.7)$ & $87(6.8)$ & $78(5.3)$ & $85(4.9)$ & 7.6 \\
\hline 47 & Imazalil & $52(1.8)$ & $68(1.0)$ & $62(4.3)$ & $65(1.7)$ & $49(3.2)$ & $64(0.8)$ & 3.8 \\
\hline 48 & Indoxacarb & $97(3.5)$ & $101(4.7)$ & $105(5.1)$ & $103(1.4)$ & $95(2.6)$ & $103(3.2)$ & 4.9 \\
\hline 49 & Imidacloprid & $80(10.6)$ & $88(8.9)$ & $73(13.2)$ & $76(9.7)$ & $72(11.7)$ & $81(10.9)$ & 12.1 \\
\hline 50 & Iprobenphos & $84(11.2)$ & $94(3.6)$ & $91(10.8)$ & $86(4.8)$ & $90(9.7)$ & $83(6.1)$ & 9.0 \\
\hline 51 & Isazophos & $94(2.1)$ & $98(4.3)$ & $89(0.9)$ & $93(4.3)$ & $92(2.1)$ & $95(3.7)$ & 4.8 \\
\hline 52 & Isoproturon & $90(1.5)$ & $95(0.5)$ & $82(2.5)$ & $89(1.5)$ & $83(3.6)$ & $82(2.4)$ & 3.7 \\
\hline 53 & Kadethrin & $95(1.1)$ & $95(4.2)$ & $90(5.3)$ & $103(2.6)$ & $94(4.8)$ & $91(7.9)$ & 8.5 \\
\hline 54 & Linuron & $93(16.7)$ & $96(11.2)$ & $99(7.9)$ & $104(9.4)$ & $94(10.5)$ & $93(7.3)$ & 13.4 \\
\hline 55 & Malathion & $100(8.5)$ & $99(4.7)$ & $97(8.6)$ & $102(7.3)$ & $97(9.5)$ & $99(3.7)$ & 7.8 \\
\hline 56 & Mefenacet & $90(3.7)$ & $95(5.8)$ & $91(3.6)$ & $88(2.1)$ & $92(6.9)$ & $91(9.4)$ & 6.6 \\
\hline 57 & Metaflumizone & $73(7.8)$ & $79(8.1)$ & $83(5.9)$ & $89(10.5)$ & $73(8.6)$ & $83(4.8)$ & 7.9 \\
\hline 58 & Metalaxyl & $98(2.1)$ & $96(3.2)$ & $87(2.8)$ & $84(3.7)$ & $80(3.8)$ & $85(2.6)$ & 4.3 \\
\hline
\end{tabular}


Table 1. continued

\begin{tabular}{|c|c|c|c|c|c|c|c|c|}
\hline \multirow[b]{3}{*}{ number } & \multirow[b]{3}{*}{ pesticide } & \multicolumn{6}{|c|}{ level of fortification, $\mu \mathrm{g} \mathrm{kg}^{-1}$} & \multirow[b]{3}{*}{ interday RSD,\% } \\
\hline & & \multicolumn{2}{|c|}{ black tea } & \multicolumn{2}{|c|}{ green tea } & \multicolumn{2}{|c|}{ oolong tea } & \\
\hline & & 10 & 50 & 10 & 50 & 10 & 50 & \\
\hline 59 & Metazachlor & $91(8.5)$ & $97(6.3)$ & $83(5.8)$ & $80(4.7)$ & $94(5.5)$ & $96(6.3)$ & 5.9 \\
\hline 60 & Methabenzthiazuron & $94(6.8)$ & $97(5.9)$ & $80(4.7)$ & $89(5.1)$ & $92(8.5)$ & $97(4.3)$ & 8.9 \\
\hline 61 & Methacrifos & $91(7.8)$ & $97(8.3)$ & $94(10.5)$ & $81(8.4)$ & $90(9.7)$ & $97(9.5)$ & 10.8 \\
\hline 62 & Methidathion & $90(8.5)$ & $95(4.7)$ & $82(5.9)$ & $79(6.8)$ & $92(9.6)$ & $89(5.7)$ & 9.6 \\
\hline 63 & Methoxyfenozide & $84(7.9)$ & $97(9.5)$ & $89(8.3)$ & $80(6.4)$ & $83(7.9)$ & $95(7.3)$ & 9.5 \\
\hline 64 & Metobromuron & $90(6.4)$ & $94(7.8)$ & $88(10.5)$ & $93(4.6)$ & $90(9.5)$ & $95(3.6)$ & 8.9 \\
\hline 65 & Metolachlor & $95(2.5)$ & $95(1.9)$ & $92(5.3)$ & $102(5.8)$ & $92(2.5)$ & $97(3.1)$ & 6.3 \\
\hline 66 & Metolcarb & $72(8.5)$ & $81(6.3)$ & $74(9.0)$ & $71(6.6)$ & $69(6.3)$ & $74(6.9)$ & 9.1 \\
\hline 67 & Metoxuron & $73(8.6)$ & $76(4.3)$ & $70(6.5)$ & $77(6.8)$ & $72(8.4)$ & $70(3.2)$ & 7.7 \\
\hline 68 & Monalide & $89(2.1)$ & $95(7.6)$ & $96(4.6)$ & $99(5.7)$ & $94(2.6)$ & $92(6.7)$ & 8.3 \\
\hline 69 & Monocrotophos & $88(11.5)$ & $85(6.9)$ & $87(13.6)$ & $77(10.5)$ & $87(13.5)$ & 89 (11.3) & 13.2 \\
\hline 70 & Monolinuron & $83(5.3)$ & $91(4.7)$ & $85(6.8)$ & $89(5.8)$ & $83(3.1)$ & $89(6.3)$ & 8.6 \\
\hline 71 & Myclobutanil & $99(3.6)$ & $101(2.5)$ & $89(6.8)$ & $99(6.4)$ & $95(5.7)$ & $98(5.0)$ & 7.3 \\
\hline 72 & Napropamide & $90(14.6)$ & $96(12.5)$ & $93(15.0)$ & $91(10.6)$ & $83(11.7)$ & $90(16.7)$ & 11.6 \\
\hline 73 & Nitenpyram & $86(6.4)$ & $82(5.8)$ & $75(8.4)$ & $83(6.0)$ & $74(9.1)$ & $72(6.6)$ & 8.6 \\
\hline 74 & Penconazole & $87(2.5)$ & $92(3.8)$ & $91(4.6)$ & $93(1.5)$ & $99(2.0)$ & $90(4.8)$ & 5.8 \\
\hline 75 & Pendimethalin & $44(0.8)$ & $46(4.3)$ & $40(3.5)$ & $48(6.2)$ & $33(4.4)$ & $37(5.0)$ & 7.3 \\
\hline 76 & Pethoxamid & $87(1.1)$ & $95(3.2)$ & $92(2.8)$ & $89(3.6)$ & $84(3.5)$ & $91(2.1)$ & 4.5 \\
\hline 77 & Phorate sulfone & $102(6.3)$ & $99(7.2)$ & $97(5.8)$ & $100(4.9)$ & $105(5.0)$ & $94(6.3)$ & 7.9 \\
\hline 78 & Phosalone & $88(3.5)$ & $91(6.1)$ & $85(6.8)$ & $93(9.3)$ & $80(3.6)$ & $89(3.9)$ & 8.0 \\
\hline 79 & Phosmet & $78(5.6)$ & $74(3.6)$ & $72(6.2)$ & $71(5.8)$ & $70(6.4)$ & $73(7.6)$ & 6.9 \\
\hline 80 & Phosphamidon & $84(5.8)$ & $82(8.7)$ & $80(5.3)$ & $85(2.0)$ & $82(8.3)$ & $80(6.9)$ & 7.3 \\
\hline 81 & Phoxim & $91(3.7)$ & $94(0.8)$ & $85(4.6)$ & $94(6.1)$ & $90(0.6)$ & $97(4.5)$ & 5.5 \\
\hline 82 & Pirimicarb & $83(0.6)$ & $84(3.5)$ & $82(6.3)$ & $79(3.5)$ & $75(2.4)$ & $77(5.1)$ & 6.0 \\
\hline 83 & Pirimiphos-ethyl & $92(1.3)$ & $90(2.7)$ & $88(3.6)$ & $91(5.2)$ & $81(4.2)$ & $85(4.0)$ & 5.4 \\
\hline 84 & Pirimiphos-methyl & $93(3.2)$ & $91(6.3)$ & $92(4.6)$ & $95(5.3)$ & $82(5.7)$ & $83(6.6)$ & 7.1 \\
\hline 85 & Pretilachlor & $96(8.5)$ & $95(7.5)$ & $100(9.6)$ & $102(4.3)$ & $97(10.5)$ & $92(10.9)$ & 9.4 \\
\hline 86 & Prochloraz & $89(7.9)$ & $93(6.3)$ & $88(9.7)$ & $82(10.0)$ & $83(6.6)$ & $81(7.4)$ & 8.2 \\
\hline 87 & Profenofos & $87(10.5)$ & $89(3.6)$ & $89(5.3)$ & $93(5.7)$ & $82(7.3)$ & $82(8.1)$ & 7.2 \\
\hline 88 & Prometryn & $92(2.0)$ & $95(1.7)$ & $90(3.6)$ & $93(5.8)$ & $89(3.5)$ & $90(3.9)$ & 4.8 \\
\hline 89 & Propachlor & $89(3.6)$ & $94(6.8)$ & $81(9.5)$ & $86(4.2)$ & $83(6.9)$ & $86(11.1)$ & 9.8 \\
\hline 90 & Propanil & $88(8.7)$ & $85(10.3)$ & $88(5.7)$ & $98(8.5)$ & $87(6.8)$ & $89(3.6)$ & 8.6 \\
\hline 91 & Propargite & $79(4.4)$ & $85(3.7)$ & $72(5.1)$ & $83(6.5)$ & $78(5.3)$ & $80(4.5)$ & 5.4 \\
\hline 92 & Propazine & $89(0.4)$ & $95(4.6)$ & $93(2.6)$ & $99(5.3)$ & $90(6.3)$ & $98(2.7)$ & 3.6 \\
\hline 93 & Propoxur & $83(5.6)$ & $91(7.1)$ & $84(8.5)$ & $88(8.0)$ & $84(6.4)$ & $81(3.4)$ & 6.1 \\
\hline 94 & Pyraclostrobine & $79(1.3)$ & $83(5.6)$ & $88(6.7)$ & $86(3.5)$ & $79(8.3)$ & $81(2.5)$ & 7.1 \\
\hline 95 & Pyridaben & $70(3.6)$ & $73(3.1)$ & $73(7.8)$ & $66(4.4)$ & $65(2.0)$ & $69(4.7)$ & 5.6 \\
\hline 96 & Pyrimethanil & $71(1.6)$ & $75(2.6)$ & $76(3.1)$ & $78(5.2)$ & $70(4.3)$ & $73(4.8)$ & 6.3 \\
\hline 97 & Quinalphos & $90(0.7)$ & $94(3.6)$ & $85(4.3)$ & $95(5.2)$ & $88(4.3)$ & $94(4.8)$ & 3.7 \\
\hline 98 & Rotenone & $80(6.7)$ & $73(3.6)$ & $78(6.2)$ & $82(5.4)$ & $80(7.1)$ & $82(7.7)$ & 8.6 \\
\hline 99 & Secbumeton & $91(3.2)$ & $94(3.0)$ & $82(5.3)$ & $87(2.7)$ & $81(2.8)$ & $83(6.1)$ & 6.7 \\
\hline 100 & Simazine & $89(4.1)$ & $88(6.2)$ & $87(5.8)$ & $92(5.4)$ & $83(3.1)$ & $85(3.9)$ & 5.7 \\
\hline 101 & Simetryn & $83(6.2)$ & $80(2.9)$ & $77(5.5)$ & $72(6.0)$ & $75(2.1)$ & $74(4.2)$ & 6.7 \\
\hline 102 & Spirodiclofen & $53(8.6)$ & $50(4.6)$ & $55(10.5)$ & $58(7.8)$ & $53(11.6)$ & $55(9.5)$ & 9.9 \\
\hline 103 & Spirotetramat & $53(7.9)$ & $58(6.3)$ & $53(11.6)$ & $61(8.4)$ & $55(6.4)$ & $52(7.7)$ & 8.5 \\
\hline 104 & Sulfotepp & $91(3.2)$ & $96(2.2)$ & $94(1.9)$ & $92(4.7)$ & $90(3.2)$ & $93(4.4)$ & 7.1 \\
\hline 105 & Sumithrin & $72(9.5)$ & $74(6.7)$ & $70(5.6)$ & $74(7.3)$ & $66(6.9)$ & $73(7.4)$ & 9.1 \\
\hline 106 & Tebufenozide & $94(13.6)$ & $96(8.4)$ & $91(9.6)$ & $90(12.6)$ & $95(11.9)$ & $92(8.9)$ & 9.3 \\
\hline 107 & Temephos & $91(6.7)$ & $94(7.1)$ & $95(6.9)$ & $107(4.3)$ & $92(6.8)$ & $89(5.6)$ & 7.5 \\
\hline 108 & Tetrachlorvinphose & $96(8.9)$ & $98(7.4)$ & $98(9.3)$ & $104(6.7)$ & $97(5.5)$ & $95(6.8)$ & 8.5 \\
\hline 109 & Tetramethrin & $83(6.7)$ & $92(8.3)$ & $95(7.3)$ & $88(5.6)$ & $91(8.3)$ & $94(5.7)$ & 9.2 \\
\hline 110 & Thiacloprid & $71(9.5)$ & $78(7.8)$ & $70(6.9)$ & $73(6.3)$ & $67(8.1)$ & $69(5.2)$ & 9.3 \\
\hline 111 & Thiodicarb & $92(5.7)$ & $89(7.5)$ & $80(6.3)$ & $78(6.9)$ & $83(10.6)$ & $82(8.5)$ & 8.7 \\
\hline 112 & Thiophanate & $90(8.7)$ & $95(7.4)$ & $90(9.1)$ & $87(11.6)$ & $95(5.8)$ & $92(7.3)$ & 8.1 \\
\hline 113 & Thiophanate-methyl & $81(9.8)$ & $84(6.7)$ & $84(7.5)$ & $82(11.3)$ & $76(8.9)$ & $78(9.3)$ & 10.8 \\
\hline 114 & Triadimefon & $98(6.5)$ & $101(7.5)$ & $97(8.2)$ & $101(3.8)$ & $100(6.7)$ & $98(5.8)$ & 9.0 \\
\hline 115 & Triazophos & $93(1.7)$ & $97(1.8)$ & $97(4.3)$ & $96(2.5)$ & $96(4.8)$ & $95(5.8)$ & 6.3 \\
\hline 116 & Trichlorfon & $75(15.6)$ & $78(11.8)$ & $82(13.4)$ & $88(14.1)$ & $83(9.5)$ & $74(11.8)$ & 12.7 \\
\hline
\end{tabular}


Table 1. continued

\begin{tabular}{|c|c|c|c|c|c|c|c|c|}
\hline \multirow[b]{3}{*}{ number } & \multirow[b]{3}{*}{ pesticide } & \multicolumn{6}{|c|}{ level of fortification, $\mu \mathrm{g} \mathrm{kg}^{-1}$} & \multirow[b]{3}{*}{ interday RSD,\% } \\
\hline & & \multicolumn{2}{|c|}{ black tea } & \multicolumn{2}{|c|}{ green tea } & \multicolumn{2}{|c|}{ oolong tea } & \\
\hline & & 10 & 50 & 10 & 50 & 10 & 50 & \\
\hline 117 & Tricyclazole & $53(3.7)$ & $53(2.7)$ & $43(6.3)$ & $48(3.4)$ & $45(3.0)$ & $42(5.7)$ & 7.5 \\
\hline
\end{tabular}

${ }^{a}$ Intraday precision, expressed as RSD (\%), is given in brackets $(n=5)$ and interday precision (RSD \%) was evaluated using spiked green tea samples at $50 \mu \mathrm{g} \mathrm{kg}^{-1}$ during 5 consecutive days. ${ }^{b}$ Fortification level at $20 \mu \mathrm{g} \mathrm{kg}^{-1}$.

$1-200 \mu \mathrm{g} \mathrm{L}^{-1}$ (equal to $8-800 \mu \mathrm{g} \mathrm{kg}^{-1}$ for tea samples). Matrix effect is calculated as follows:

$$
\operatorname{ME}(\%)=\left[\left(\frac{\text { Slope of matrix matched calibration curve }}{\text { Slope of solvent calibration curve }}\right)-1\right]
$$$$
\times 100
$$

The method was validated according to the international SANTE guidelines SANTE/11945/2015. ${ }^{26}$ Representative matrices, including green tea (unfermented), black tea (fermented), and oolong tea (semifermented), were used for validation. Linearity was evaluated using matrix matched standard solution in the range of $1-200 \mu \mathrm{g} \mathrm{L}^{-1}$ (equal to $4-800 \mu \mathrm{g} \mathrm{kg}^{-1}$ for tea sample). Accuracy was measured in terms of recoveries obtained by five blank samples fortified at 10 and $50 \mu \mathrm{g} \mathrm{kg}^{-1}$ of the respective analytes. The lowest fortification level was chosen based on the lowest maximum residue levels (MRLs) which were generally set up at $10 \mu \mathrm{g} \mathrm{kg}^{-1}$. Precision was evaluated as intraday and interday repeatability, expressed as relative standard deviation (RSD). Limits of detection (LODs) were obtained by injecting fortified samples at $0.5,1.0,2.0,5.0$, and $10.0 \mu \mathrm{g} \mathrm{kg}^{-1}$. Limits of quantification (LOQs) were determined by analyzing minimum fortified samples that provided suitable recovery in the range of $70-130 \%$ and RSDs within $20 \%$.

\section{RESULTS AND DISCUSSION}

3.1. Establishment of Compound Database for Qualification and Quantification. To develop a database of target compounds, individual standard solutions for 117 pesticides at $50 \mu \mathrm{g} \mathrm{L}^{-1}$ were injected into the UPLC-Q-OrbitrapMS, and the MS acquisition in positive was performed with and without fragmentation in the HCD collision cell. Table S-1 shows the accurate mass, retention time, and fragmented ions obtained from full scan/dd-MS ${ }^{2}$ mode. Mutual interference was not observed for 113 pesticides based on chromatographic retention time window $\pm 0.2 \mathrm{~min}$ and mass error $\pm 5 \mathrm{ppm}$. For full scan mode, the compounds with the same mass (isomers) are hardly distinguished because they have the same molecular mass weight. As shown in Figure 1, quinalphos and phoxim have the same chemical formula $\mathrm{C}_{12} \mathrm{H}_{15} \mathrm{~N}_{2} \mathrm{O}_{3} \mathrm{PS}$ with the theoretical mass $m / z$ at $299.0613 \mathrm{Da}$ (experimental mass at $\mathrm{m} / z$ 299.0607 Da), and the retention time deviation was less than $0.1 \mathrm{~min}$. In this case, dd-MS ${ }^{2}$ monitoring mode is necessary for obtaining fragmented ions to identify quinalphos and phoxim. In the HCD collision cell, the protonated molecular ion $[\mathrm{M}+\mathrm{H}]^{+}$of quinalphos was fragmented into the product ions at $\mathrm{m} / \mathrm{z} 147.0550$ and 163.0326 , while the fragmented product ions at $m / z 129.0448$ and 114.9609 were obtained from the phoxim protonated molecular ion. Besides quinalphos and phoxim, the isomers pretilachlor and metolachlor have the same accurate mass and similar retention time and different fragmented ions of protonated molecular ion, which is shown in Figure S-1. Therefore, UPLC-Q-Orbitrap MS was performed with full scan monitoring mode for identification and quantification, and full scan/dd-MS ${ }^{2}$ was complementary for identification of the compounds with the same chemical formula and molecular weight.

In this study, interference from the tea matrix was investigated. No interference from the matrices was found for any of the pesticides, except allethrin, when retention time window set at $0.5 \mathrm{~min}$. As shown in Figure 2, three chromatographic peaks (extracted ion $m / z$ 303.1952) obtained from blank tea sample could result in the interference with allethrin (mass error at $5 \mathrm{ppm}$ ). To evaluate retention time tolerance, 20 successive injections of a mixture of green tea matrix matched calibration solution at $50 \mu \mathrm{g} \mathrm{L}^{-1}$ were carried out. The results showed that retention time deviations for all pesticides were less than $0.15 \mathrm{~min}$. Therefore, the retention time window set at $0.2 \mathrm{~min}$ in this study, and none of the 117 pesticides interfered with the tea matrix.

3.2. Determination of Extraction Efficiency of Pesticide Residues in Incurred Tea Samples Using Different Extraction Techniques. In order to evaluate the extraction efficiency of multi pesticide residues, we choose different types of pesticides, which have a wide range of water solubility $\left(0.48-39800 \mathrm{mg} / \mathrm{L}, 20^{\circ} \mathrm{C}\right)$ and octanol-water ratio ( $\log K_{\mathrm{ow}}$ $0.8-6.37)$. The effect of extraction technique on detected concentrations of 11 pesticide residues in 10 incurred tea samples was investigated. First, the effect of the additional water on extraction efficiency was investigated. Figure 3 illustrates that all detected concentrations of 11 incurred pesticides increased, ranging from 1.1 to 18.6 times, when tea samples were allowed to be immersed in boiling water prior to acetonitrile extraction, in comparison with the use of acetonitrile as extraction solvent without soaking in boiling water. The enhancement ratio seems negatively correlated with octanol-water partition coefficient $\left(\log K_{\mathrm{ow}}\right)$. Second, the effect of soaking by boiling water and room-temperature water on extraction efficiency was investigated. As shown in Table S-2, the detected concentrations obtained from soaking in water (at $20-25^{\circ} \mathrm{C}$ ) (E5) were less than $3.6-14.9 \%$ of the average values obtained from six extraction procedures. The results illustrated that boiling water was helpful for acetonitrile to permeate tea leaves and improve extraction efficiency of pesticides. Finally, different extraction techniques were compared and the results were shown in Table S-2. Statistical analysis based on SPSS LSD test showed that there was no significant difference of detected concentrations of 11 pesticides in 10 incurred tea samples using five extraction techniques, e.g., standing overnight (about $12 \mathrm{~h}$ ) (E1), vortexed for $2 \mathrm{~min}$ (E2), rotational oscillation at $300 \mathrm{rpm}$ for $10 \mathrm{~min}$ (E3) or $20 \mathrm{~min}$ (E4), and homogenization with blend probe (E6). The results obtained from E6 were slightly higher than from other extraction techniques. The extraction procedure is one of the key points for high throughput sample preparation and it requires simultaneous processing of multiple samples. For homogenization with one blend probe, it is inconvenient, slow, laborious, and potentially hazardous due to 


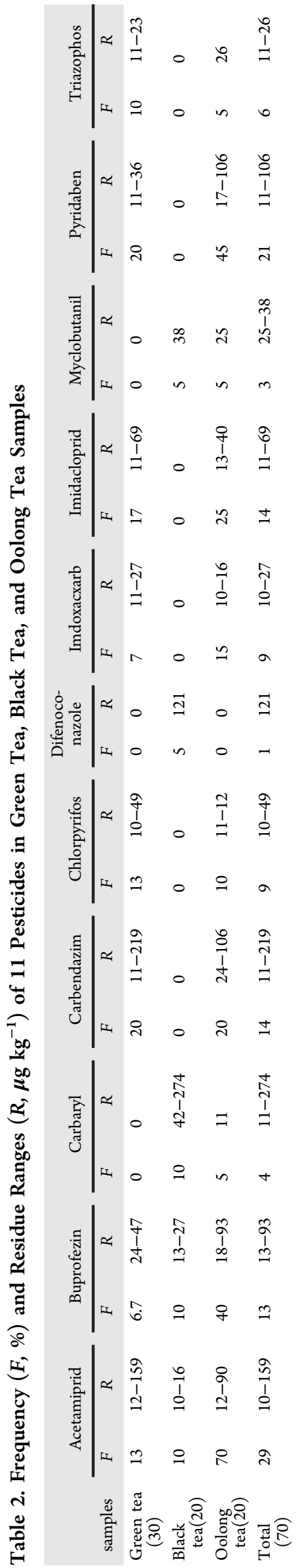

its extensive and problematic cleaning steps. ${ }^{27}$ For high throughput sample analysis, extraction procedures E1 (stand by for overnight) and E3 (rotational oscillation for $10 \mathrm{~min}$ ) were proposed, while extraction procedures E2 and E3 were suitable for quick sample preparation.

3.3. Optimization of IS-D-SPE Cleanup Procedure. IS-D-SPE is a dispersive solid extraction (D-SPE) sample preparation technique using a syringe containing adsorbents for cleanup, which does not need centrifugation and therefore takes less time for sample preparation. ${ }^{28-31}$ Several dispersive adsorbents (PSA, C18, GCB, SAX, florisil, carbon nanotubes) have been previously reported for removal of tea matrix as D-SPE adsorbents. ${ }^{8,32-38}$ In this study, PSA, C18, and MWCNTs were prepared for cleanup in the mixture of IS-D-SPE adsorbents, and each mass of adsorbents was optimized. To evaluate the recovery and cleanup effects, blank extracts spiked with 117 pesticides at $25 \mu \mathrm{g} \mathrm{L}{ }^{-1}$ were investigated using different adsorbents. All pesticides could obtain good recovery in the range of $90-110 \%$, and the color density of extracts had little change when PSA and C18 were individually used as IS-D-SPE adsorbents with the amount of $0-200 \mathrm{mg}$, which indicated that both adsorbents had little adsorption capacity of 117 pesticides, as well as pigments or other color substances. MWCNTs, as a carbon-based nanomaterial, has been reported as an excellent adsorbent for the removal of agricultural sample matrix owing to the large surface area and structural characteristics. ${ }^{39-41}$ Color density of tea extracts became lighter when the mass of MWCNTs increased from 0 to $50 \mathrm{mg}$. In consideration of the adsorptivity of MWCNTs for pesticides, mixture adsorbents of $200 \mathrm{mg}$ PSA, $100 \mathrm{mg} \mathrm{C18}$, and MWCNTs with the mass of 0-50 mg were optimized. As shown in Figure 4, satisfactory recoveries in the range of $80-120 \%$ were obtained for 109 pesticides when $50 \mathrm{mg}$ MWCNTs was used. Eight pesticides (e.g., chlorbenzuron, clofentezine, diflufenican, pendimethalin, phosmet, profenofos, rotenone, and spirodiclofen) seriously lost their recovery with the MWCNTs cleanup treatment. By comparison, $15 \mathrm{mg}$ of MWCNTs was proposed because higher recovery of chlorbenzuron, clofentezinethe, and phosmet weas observed. Finally, a mixture of $200 \mathrm{mg}$ PSA, $100 \mathrm{mg} \mathrm{C18}$, and $15 \mathrm{mg}$ MWCNTs was used as IS-D-SPE adsorbent.

3.4. Method Validation. The obtained data of matrix effects are shown in Figure 5. Ion suppression with matrix effect value below 0 was observed for most analytical pesticides in black tea, green tea, and oolong tea. Pesticides with significant matrix effect below $-20 \%$ from black tea, green tea, and oolong tea accounted for the percentage of $11 \%, 21 \%$, and $26 \%$, respectively. Generally, matrix suppression was stronger for unfermented tea (green tea) and semifermented tea (oolong tea) than fermented tea (black tea). Eleven pesticides, e.g., carbendazim (51-70\%), dichlorvos (22-90\%), dimethoate (20-30\%), flonicamid (81-85\%), methacrifos (22-36\%), monocrotophos (49-51\%), sebuthylazine (27\%), simetryn (54-55\%), spirotetramat $(31-35 \%)$, thionphanate-methyl (43-66\%), and trichlorfon $(41-52 \%)$, showed strong ion suppression below $-20 \%$. Therefore, matrix matched calibration solutions were employed to establish the calibrated curves for each pesticide. Good linearity was achieved for all 117 pesticides with correlation coefficients $\left(R^{2}\right)$ higher than 0.991 (see Table S-3).

Accuracy was evaluated in terms of recovery at spiked levels of $10 \mu \mathrm{g} \mathrm{kg}^{-1}$ (20 $\mu \mathrm{g} \mathrm{kg}^{-1}$ for dimetachlone) and $50 \mu \mathrm{g} \mathrm{kg}^{-1}$, shown in Table 1. Except for 12 pesticides, e.g., chlorbenzuron (21-31\%), chlorfluazuron (61-71\%), chlorotoluron (46-52\%), clofentezine (22-34\%), diflubenzuron (40-57\%), diflufenican 


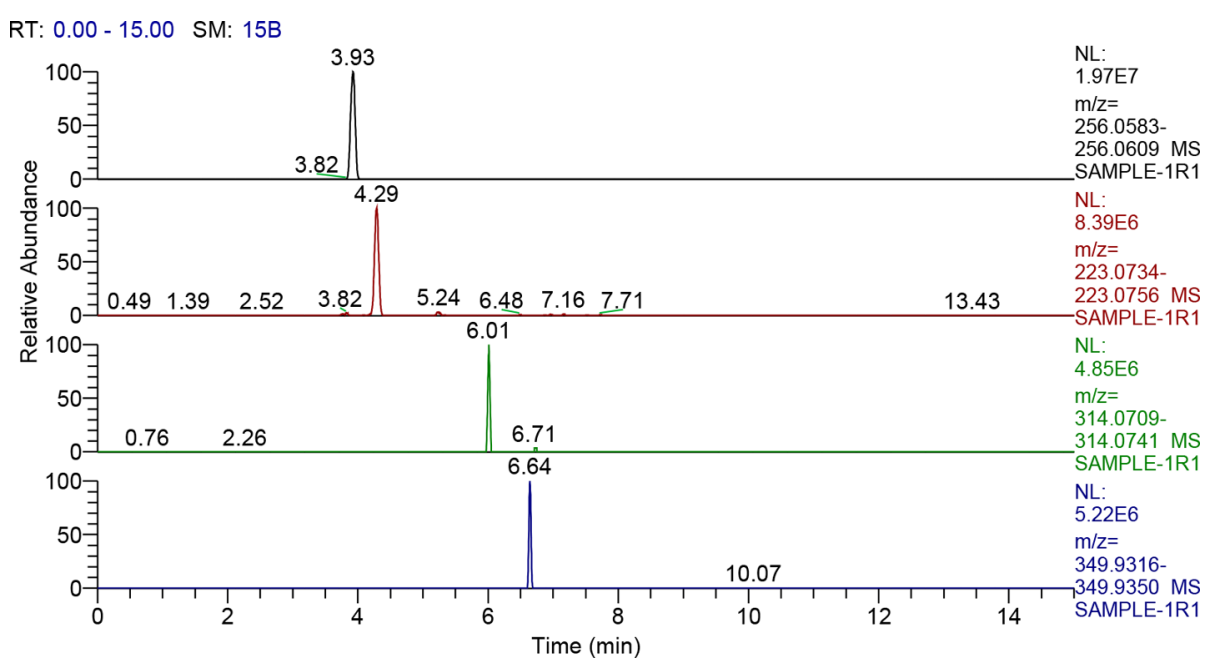

Figure 6. Extracted ion chromatograms of imidacloprid $(\mathrm{m} / z 256.0596)$ at $79 \mu \mathrm{g} \mathrm{kg}^{-1}$, acetamiprid $(\mathrm{m} / z 223.0745)$ at $5 \mu \mathrm{g} \mathrm{kg}$, triazophos $\left(\mathrm{m} / z\right.$ 314.0725) at $0.1 \mu \mathrm{g} \mathrm{kg}^{-1}$, and chlorpyrifos $(\mathrm{m} / z 349.9333)$ at $0.3 \mu \mathrm{g} \mathrm{kg}^{-1}$ detected in an oolong tea sample. (note: Quantification by single point calibration using matrix matched calibration solution at $1 \mu \mathrm{g} \mathrm{L}^{-1}$.)

(43-64\%), fenazaquin (49-61\%), imazalil (49-68\%), pendimethalin (33-48\%), spirodiclofen $(50-58 \%)$, spirotetramat $(52-61 \%)$, and tricyclazole $(42-53 \%)$, recoveries were in the range of $70-110 \%$. For different kinds of tea samples, there is little difference in recovery among black tea, green tea, and oolong tea. Physicochemical properties of analytical pesticides were responsible for the loss of recovery, especially due to the adsorption of MWCNTs during IS-D-SPE cleanup.

Precision was measured employing intraday repeatability and interday reproducibility testing. Intraday repeatability was evaluated at the fortified levels of $10 \mu \mathrm{g} \mathrm{kg}^{-1}$ and $50 \mu \mathrm{g} \mathrm{kg}^{-1}$ during the same day. In this case, the repeatability, expressed as RSDs, was less than $17 \%$ (see Table 1) for all analytical pesticides. Interday reproducibility was evaluated analyzing five blank green tea samples spiked with $50 \mu \mathrm{g} \mathrm{kg}^{-1}$ of 117 pesticides during 5 consecutive days. Interday RSDs for all analytical pesticides were below $18 \%$.

LODs were estimated analyzing three blank samples spiked at $0.5,1.0,2.0,5.0$, and $10.0 \mu \mathrm{g} \mathrm{kg}^{-1}$. They are defined as the minimum concentration at which the accurate mass error was $\pm 5 \mathrm{ppm},{ }^{42}$ and LODs were less than or equal to $5 \mu \mathrm{g} \mathrm{kg}^{-1}$ for all pesticides except dimetachlone at $10 \mu \mathrm{g} \mathrm{kg}^{-1}$ (see Table S-3). According to accuracy and precision measurement, LOQs were $10 \mu \mathrm{g} \mathrm{kg}^{-1}$ for 104 pesticides which provided recoveries between $70 \%$ and $130 \%$, and RSDs below $20 \%$. LOQs of dimetachlone were $20 \mu \mathrm{g} \mathrm{kg}^{-1}$ due to poor sensitivity. Although low recoveries for 9 pesticides, including chlorfluazuron, chlorotoluron, diflubenzuron, diflufenican, fenazaquin, imazalil, spirodiclofen, spirotetramat, and tricyclazole, were in the range of $40-70 \%$, these pesticides could be quantified using this method according to European Council No SANTE/11945/2015, ${ }^{26}$ where the document shows that a mean recovery below $70 \%$ may be acceptable when the method is quite consistent (with a good precision). Due to the low recovery below $40 \%$, this method was not suitable for quantification of chlorbenzuron, clofentezine, and pendimethalin in tea.

3.5. Real Sample Analysis. A total of 70 tea samples ( 30 green tea samples, 20 black tea samples, and 20 oolong tea samples) purchased from local markets in Zhejiang province were analyzed using this proposed UPLC-Q-Orbitrap-MS method. For each batch of less than 20 tea samples, quality control samples spiked with 117 pesticides at 10 and $50 \mu \mathrm{g} \mathrm{kg}^{-1}$ were analyzed to evaluate the characteristics of the analytical method. Target analyte identification was based on retention time window $\pm 0.2 \mathrm{~min}$ and mass accuracy $\pm 5 \mathrm{ppm}$. To confirm positive samples, characteristic fragments and isotopic pattern were monitored using UPLC-Q-Orbitrap-MS in full scan/dd-MS ${ }^{2}$ mode. Table 2 shows the frequency and concentrations of 11 detected pesticides, including acetamiprid, buprofezin, carbaryl, carbendazim, chlorpyrifos, difenoconazole, indoxacarb, imidacloprid, myclobutail, pyridaben, and triazophos. Higher frequencies above $10 \%$ occurred for two neonicotinoid pesticides (acetamiprid and imidacloprid), buprofezin, carbedazim, and pyredaben. Multi residues containing over 3 pesticides in the same sample were observed for green tea (9.2\%), black tea $(2.8 \%)$, and oolong tea (15.9\%). Figure 6 shows an oolong tea containing actamiprid $\left(5 \mu \mathrm{g} \mathrm{kg}^{-1}\right)$, chlorpyrifos $\left(0.3 \mu \mathrm{g} \mathrm{kg}^{-1}\right)$, imidacloprid $\left(79 \mu \mathrm{g} \mathrm{kg}^{-1}\right)$, and triazophos $\left(0.1 \mu \mathrm{g} \mathrm{kg}^{-1}\right)$. None of the 117 pesticides was higher than EU maximum residue levels (MRLs), CAC MRLs and China MRLs.

\section{CONCLUSIONS}

A high throughput method based on in-syringe dispersive solid phase extraction and UPLC-Q-Orbitrap MS was developed for rapid analysis of multiple pesticide residues in green tea, black tea, and oolong tea. A mixture of adsorbents containing PSA, C18, and MWCNTs was used as IS-D-SPE adsorbents to reduce tea matrices. UPLC-Q-Orbitrap-MS at full scan monitoring mode provided high method selectivity, accuracy, and precision. Full mass/dd-MS ${ }^{2}$ monitoring mode is necessary to identify the compounds with the same chemical formula. Matrix suppression was more serious for unfermented tea and semifermented tea than fermented tea. This newly developed method allows high sample preparation thanks to its simplicity and effectiveness. Compared with previous analytical methods, the advantages of this proposed method are less time-consuming, solvent-consuming, lower cost, and higher throughout analysis. Therefore, this method is suitable for routine analysis and identification of multiple pesticides in various types of tea samples. This method was applied to analyze 70 tea samples. High frequency of two neonicotinoid pesticides acetamiprid and imidacloprid, buprofezin, carbendazim, and pyredaben was found. 


\section{ASSOCIATED CONTENT}

\section{S Supporting Information}

The Supporting Information is available free of charge on the ACS Publications website at DOI: 10.1021/acsomega.7b00863.

Compound database of 117 pesticides obtained from UPLC-Q-Orbitrap MS. The effect of extraction mode on the analytical results of 11 pesticides in 10 incurred tea samples. Linearity, LODs, and LOQs of 117 pesticides. Extracted ion chromatograms of metalachlor and prelachlor. (PDF)

\section{AUTHOR INFORMATION}

\section{Corresponding Authors}

*E-mail: Liuxin@mail.tricaas.com (X. Liu).

*E-mail: Lchy@mail.tricaas.com (C. Lu).

\section{ORCID $\odot$}

Chengyin Lu: 0000-0002-5794-864X

\section{Notes}

The authors declare no competing financial interest.

\section{ACKNOWLEDGMENTS}

This work was supported by the earmarked fund for National Natural Funding (31671941), Innovative Research Team in Chinese Academy of Agricultural Sciences (CAAS-ASTIP-2014TRICAAS), Public Welfare and Technique Project of Zhejiang province (2017C32059), Modern Agro-Industry Technology Research System (CARS-23), and Zhejiang Provincial Natural Science Foundation of China (LY15C200019).

\section{REFERENCES}

(1) Sharangi, A. B. Medicinal and therapeutic potentialities of tea (Camellia sinensis L.)-A review. Food Res. Int. 2009, 42, 529-535.

(2) Xiao, Q. Tea diseases and insect pest. Chinese Tea 2014, 3, 16-17 (In Chinese).

(3) El-Aty, A. M. A.; Choi, J.; Rahman, M. M.; Kim, S.; Tosun, A.; Shim, J. Residues and contaminants in tea and tea infusion: a review. Food Addit. Contam., Part A 2014, 31 (11), 1794-1804.

(4) European Union. Pesticide residues and maximum residue levels [DB/OL]; http://ec.europa.eu/food/plant/pesticides/eu-pesticidesdatabase/public/?event=download.MRL [2016/06/05].

(5) The Japan Food Chemical Research Foundation. Maximum Residue Limits (MRLs) of Agricultural Chemicals in Foods [DB/OL]; http://www.ffcr.or.jp/zaidan/FFCRHOME.nsf/pages/MRLs-p [2017/05/16].

(6) National food safety standard, GB 2763-2016, Maximum residue limits for pesticides in food.

(7) Zhang, X.; Mobley, N.; Zhang, J.; Zheng, X.; Lu, L.; Ragin, O.; Smith, C. J. Analysis of Agricultural residues on tea using d-SPE sample preparation with GC-NCI-MS and UHPLC-MS/MS. J. Agric. Food Chem. 2010, 58, 11553-11560.

(8) Lozano, A.; Rajski, Ł.; Belmonte-Valles, N.; Uclés, A.; Uclés, S.; Mezcua, M.; Fernández-Alba, A. R. Pesticide analysis in teas and chamomile by liquid chromatography and gas chromatography tandem mass spectrometry using a modified QuEChERS method: Validation and pilot survey in real samples. J. Chromatogr A 2012, 1268, 109122.

(9) Masiá, A.; Suarez-Varela, M. M.; Llopis-Gonzalez, A.; Picó, Y. Determination of pesticides and veterinary drug residues in food by liquid chromatography-mass spectrometry: A review. Anal. Chim. Acta 2016, 936, 40-61.

(10) Pang, G.; Fan, C.; Zhang, F.; Li, Y.; Chang, Q.; Gao, Y.; Liu, Y.; Li, Z.; Wang, Q.; Hu, X.; Liang, P. High-throughput GC/MS and HPLC/MS/MS technique for the multiclass, multiresidue determi- nation of 653 pesticides and chemical pollutants in tea. J. AOAC Int 2011, 94 (4), 1253-1296.

(11) Kaufmann, A.; Butcher, P.; Maden, K.; Walker, S.; Widmer, M. Comprehensive comparison of liquid chromatography selective as provided by two types of liquid chromatography detectors (high resolution mass spectrometry and tandem mass spectrometry):" Where is the crossover point? Anal. Chim. Acta 2010, 673, 60-72.

(12) Kellmann, M.; Muenster, H.; Zomer, P.; Mol, H. Full scan MS in comprehensive qualitative and quantitative residue analysis in food and feed matrices: How much resolving power is required. J. Am. Soc. Mass Spectrom. 2009, 20, 1464-1476.

(13) Croley, T. R; White, K. D.; Callahan, J. H.; Musser, S. M. The chromatographic role in high resolution mass spectrometry for nontargeted analysis. J. Am. Soc. Mass Spectrom. 2012, 23 (9), 1569-1578.

(14) Masiá, A.; Campo, J.; Blasco, C.; Picó, Y. Ultra-high performance liquid chromatography-quadrupole time-of-flight mass spectrometry to identify contaminants in water: An insight on environmental forensics. J. Chromatogr A 2014, 1345, 86-97.

(15) Hogenboom, A. C.; van Leerdam, J. A.; de Voogt, P. Accurate mass screening and identification of emerging contaminants in environmental samples by liquid chromatography-hybrid linear ion trap orbitrap mass spectrometry. J. Chromatogr A 2009, 1216, 510519.

(16) Makarov, A. Electrostatic axially harmonic orbital trapping: A high performance technique of mass analysis. Anal. Chem. 2000, 72 (6), 1156-1162.

(17) Hu, Q.; Noll, R. J.; Li, H.; Makarov, A.; Hardman, M.; Cooks, R. G. The Orbitrap: a new mass spectrometer. J. Mass Spectrom. 2005, 40, 430-443.

(18) Makarov, A. Performance evaluation of a hybrid linear ion trap/ orbitrap mass spectrometry. Anal. Chem. 2006, 78, 2113-2121.

(19) Lommen, A.; Gerssen, A.; Oosterink, J. E.; Kools, H. J.; RuizAracama, A.; Peters, R. J. B.; Mol, H. G. J. Ultra-fast searching assists in evaluating sub-ppm mass accuracy enhancement in U-HPLC/Orbitrap MS data. Metabolomics 2011, 7, 15-24.

(20) Rajski, L.; Gómez-Ramos, M. M.; Fernández-Alba, A. R. Large pesticide multiresidue screening method by liquid chromatographyOrbitrap mass spectrometry in full scan mode applied to fruit and vegetables. J. Chromatogr A 2014, 1360, 119-127.

(21) Gómez-Pérez, M. L.; Plaza-Bolaños, P.; Romero-González, R.; Martínez-Vidal, J. L.; Garrido-Frenich, A. Comprehensive qualitative and quantitative determination of pesticides and veterinary drugs in honey using liquid chromatography-Orbitrap high resolution mass spectrometry. J. Chromatogr A 2012, 1248, 130-138.

(22) Farré, M.; Picó, Y.; Barceló, D. Application of ultra-high pressure liquid chromatography linear ion-trap orbitrap to qualitative and quantitative assessment of pesticide residues. J. Chromatogr $A$ 2014, 1328, 66-79.

(23) Jia, W.; Chu, X.; Ling, Y.; Huang, J.; Chang, J. High-throughput screening of pesticide and veterinary drug residues in baby food by liquid chromatography coupled to quadrupole Orbitrap mass spectrometry. J. Chromatogr A 2014, 1347, 122-128.

(24) Martínez-Domínguez, G.; Romero-González, R.; GarridoFrenich, A. Multi-class methodology to determine pesticides and mycotoxins in green tea and royal jelly supplements by liquid chromatography coupled to Orbitrap high resolution mass spectrometry. Food Chem. 2016, 197, 907-915.

(25) del Mar Gómez-Ramos, M.; Rajski, Ł.; Heinzen, H.; FernándezAlba, A. R. Liquid chromatography Orbitrap mass spectrometry with simultaneous full scan and tendem MS/MS for highly selective pesticide residue analysis. Anal. Bioanal. Chem. 2015, 407, 6317-6326.

(26) European Commision Health \& Consumer Protection Directorate General, SANTE/11945/2015 30 November-1 December 2015 rev. Guidance document on analytical quality control and validation procedures for pesticide residues in food and feed; .

(27) Sapozhnikova, Y.; Lehotay, S. J. Evaluation of different parameters in the extraction incurred pesticides and environmental contaminants in fish. J. Agric. Food Chem. 2015, 63 (21), 5163-5168. 
(28) Zhao, P.; Huang, B.; Li, Y.; Han, Y.; Zou, N.; Gu, K.; Li, X.; Pan, C. Rapid multiplug filtration cleanup with multiple-walled carbon nanotubes and gas chromatography-triple-quadruple mass spectrometry detection for 186 pesticide residues in tomato and tomato products. J. Agric. Food Chem. 2014, 62, 3710-3725.

(29) Tang, S.; Lin, X. H.; Li, F. Y.; Lee, H. K. In-syringe dispersive solid-phase extraction using dissolvable layered double oxide hollow spheres as sorbent followed by high-performance liquid chromatography for the determination of 11 phenols in river water. J. Chromatogr A 2014, 1373, 31-39.

(30) Yang, X.; Zhang, P.; Li, X.; Hu, L.; Gao, H.; Zhang, S.; Zhou, W.; Lu, R. Effervescence-assisted $\beta$-cyclodextrin/attapulgite composite for the in-syringe dispersive solid-phase extraction of pyrethroids in environmental water samples. Talanta 2016, 153, 353-359.

(31) García-Valverde, M. T.; Lucena, R.; Cárdenas, S.; Valcárcel, M. In-syringe dispersive micro-solid phase extraction using carbon fibres for the determination of chlorophenols in human urine by gas chromatography/mass spectrometry. J. Chromatogr A 2016, 1464, 4249.

(32) Martínez-Domínguez, G.; Nieto-García, A. J.; RomeroGonzález, R.; Frenich, A. G. Application of QuEChERS based method for the determination of pesticides in nutraceutical products (Camellia sinensis) by liquid chromatography coupled to triple quadrupole tandem mass spectrometry. Food Chem. 2015, 177, 182-190.

(33) Zhang, Y.; Xu, H. Determination of triazoles in tea samples using dispersive solid phase extraction combined with dispersive liquid-liquid microextraction followed by liquid chromatographytandem mass spectrometry. Food Anal Method 2014, 7, 189-196.

(34) Chen, G.; Cao, P.; Liu, R. A multi-residue method for fast determination of pesticides in tea by ultra performance liquid chromatography-electrospray tandem mass spectrometry combined with modified QuEChERS sample preparation procedure. Food Chem. 2011, 125, 1406-1411.

(35) Cajka, T.; Sandy, C.; Bachanova, V.; Drabova, L.; Kalachova, K.; Pulkrabova, J.; Hajslova, J. Streamlining sample preparation and gas chromatography-tandem mass spectrometry analysis of multiple pesticide residues in tea. Anal. Chim. Acta 2012, 743, 51-60.

(36) Zhao, P.; wang, L.; Jiang, Y.; Zhang, F.; Pan, C. Dispersive cleanup of acetonitrile extracts of tea samples by mixed multiwalled carbon nanotubes, primary secondary amine, and graphitized carbon black sorbents. J. Agric. Food Chem. 2012, 60 (16), 4026-4033.

(37) Sadowska-Rociek, A.; Surma, M.; Cieślik, E. Comparison of different modifications on QuEChERS sample preparation method for PAHs determination in black, green, red and white tea. Environ. Sci. Pollut. Res. 2014, 21 (2), 1326-1338.

(38) Lozowicka, B.; Ilyasova, G.; Kaczynski, P.; Jankowska, M.; Rutkowska, E.; Hrynko, I.; Mojsak, P.; Szabunko, J. Multi-residue methods for the determination of over four hundred pesticides in solid and liquid high sucrose content matrices by tandem mass spectrometry coupled with gas and liquid chromatograph. Talanta 2016, 151, 5161.

(39) Fang, G.; Min, G.; He, J.; Zhang, C.; Qian, K.; Wang, S. Multiwalled carbon nanotubes as matrix solid-phase dispersion extraction absorbents to determine 31 pesticides in agriculture sample by gas chromatography-mass spectrometry. J. Agric. Food Chem. 2009, 57, 3040-3045.

(40) Qin, Y.; Zhang, J.; He, Y.; Han, Y.; Zou, N.; Li, Y.; Chen, R.; Li, $\mathrm{X}$; Pan, C. Automated multiplug filtration cleanup for pesticide residue analyes in Kiwi fruit (Actinidia chinesis) and Kiwi juice by gas chromatography-mass spectrometry. J. Agric. Food Chem. 2016, 64, 6082-6090.

(41) Asensio-Ramos, M.; Hernández-Borges, J.; Borges-Miquel, T. M.; Rodríguez-Delgado, M. A. Evaluation of multi-walled carbon nanotubes as solid-phase extraction adsorbents of pesticides from agricultural, ornamental and forestal soils. Anal. Chim. Acta 2009, 647, $167-176$.

(42) Gómez-Pérez, M. L.; Romero-González, R.; Plaza-Bolaños, P.; Génin, E.; Vidal, J. L. M.; Frenich, A. G. Wide-scope analysis of pesticide and veterinary drug residues in meat matrices by high resolution MS: detection and identification using Exactive-Orbitrap. J. Mass Spectrom. 2014, 49, 27-36. 